\title{
FOLFOXIRI-Panitumumab Regimen
}

National Cancer Institute

\section{Source}

National Cancer Institute. FOLFOXIRI-Panitumumab Regimen. NCI Thesaurus. Code C161590.

A regimen consisting of fluorouracil, leucovorin, oxaliplatin and irinotecan (FOLFOXIRI), plus panitumumab that may be used in the treatment of certain types of colon and rectal cancers. 